\title{
Costo de criar una ternera lechera de reemplazo desde el nacimiento al parto ${ }^{1}$
}

\section{Cost of raising a replacement dairy heifer from birth to calving}

\author{
Jorge Alberto Elizondo-Salazar ${ }^{2}$, Heber Ariel Solís-Chaves ${ }^{3}$
}

\begin{abstract}
Resumen
Los reemplazos son el futuro de cualquier explotación lechera; sin embargo, antes de que comiencen a producir leche, representan un costo muy significativo y poco conocido por un número importante de productores. El objetivo del presente estudio fue determinar el costo económico asociado a la cría de reemplazos de lechería. El estudio se llevó a cabo en la Estación Experimental de Ganado Lechero Alfredo Volio Mata de la Universidad de Costa Rica, durante el año 2016. Se utilizó un total de cuarenta animales de la raza Jersey desde el nacimiento hasta el parto (veinticinco meses), agrupados en tres categorías de acuerdo con su edad: 0-3 meses, 3 a 16 meses y de 16 a 25 meses. Para determinar los costos totales dentro de cada etapa, se llevaron registros minuciosos en una hoja electrónica de todos los gastos en que se incurrió cada día y para cada animal; se consideraron todas las actividades que se llevan a cabo y que tienen que ver con alimentación, limpieza y desinfección, tratamientos veterinarios, fertilización de pasturas, mano de obra, plan sanitario y de mantenimiento, entre otros. Se determinó que el costo total para desarrollar un animal desde el nacimiento hasta el parto fue de $\phi 818360,95$ ( $\$ 1473,62)$; representando un 25,$17 ; 53,27$ y $21,56 \%$ para la etapa del nacimiento a los tres meses, de los tres meses al servicio y del servicio al parto, respectivamente. Del total de los costos, un 78,72\% correspondió a alimentación y un $6,82 \%$ a mano de obra. Dependiendo de la producción de leche y de la ganancia económica en cada litro de leche, la inversión realizada se estaría recuperando más allá de la segunda lactancia.
\end{abstract}

Palabras clave: costos de producción, economía de la producción, factores de producción, manejo del ganado, terneras.

\begin{abstract}
Dairy heifers are the future of any dairy farm; however, before they begin to produce milk, they represent a very significant cost unknown by an important number of producers. The objective of the present study was to determine the economic cost associated with the rearing of dairy replacements. The study was carried out at the dairy cattle Alfredo Volio Mata Experiment Station of the University of Costa Rica, during the year 2016. A total of forty Jersey heifers were reared from birth to calving (twenty-five months) grouped into three age categories: 0-3 months, 3 to 16 months and 16 to 25 months. In order to determine the total costs within each category, detailed records of all
\end{abstract}

1 Recibido: 26 de febrero, 2018. Aceptado: 30 de mayo, 2018. Este trabajo formó parte del proyecto de investigación inscrito en la Vicerrectoría de Investigación, No. 737-B4-222. Universidad de Costa Rica, San José, Costa Rica.

2 Universidad de Costa Rica, Facultad de Ciencias Agroalimentarias, Estación Experimental Alfredo Volio Mata e Instituto de Investigaciones Agrícolas. Cartago, Costa Rica. jorge.elizondosalazar@ucr.ac.cr (autor para correspondencia).

3 Universidad de Costa Rica, Facultad de Ciencias Agroalimentarias, Estación Experimental Alfredo Volio Mata. Cartago, Costa Rica. hebersolis1993@hotmail.com 
the expenses incurred each day for each animal were kept in an electronic spreadsheet, considering all the activities involved and related to feeding, cleaning and disinfection, veterinary treatments, pasture fertilization, workforce, health management and maintenance, among others. It was determined that the total cost for raising a replacement heifer from birth to calving was $₫ 818360.95$ (\$1473.62); representing a $25.17 ; 53.27$ and $21.56 \%$ for the stage of birth to three months, three months to service, and service to calving, respectively. Of the total costs, $78.72 \%$ corresponded to feed and $6.82 \%$ to labor. Depending on milk production and net economic return from each liter of milk produced, the investment would be recovered beyond the second lactation.

Keywords: calves, livestock management, production costs, production economics, production factors.

\section{Introducción}

La industria lechera juega un papel muy importante a nivel mundial y Costa Rica no es la excepción. En el país existen alrededor de 93017 fincas agropecuarias, de las cuales el 28,5\% tienen como actividad principal la producción de ganado vacuno (INEC, 2015). Según el VI Censo Nacional Agropecuario, para el año 2014 el país contaba con 1278817 cabezas de ganado vacuno, 42,1\% destinado a producción de carne, 32,0\% al doble propósito y 25,6\% a la producción de leche (INEC, 2015).

Para el año 2012, la producción de leche nacional generó 46147 empleos directos entre fincas e industrias lácteas, y ocupó el 74,8\% del volumen de las exportaciones del sector pecuario (Madriz, 2014). Además, la actividad lechera ha presentado un crecimiento importante en los últimos años, pasó de 953000 toneladas métricas en el 2010 a 1077000 en el 2014 (Gutiérrez, 2015). Esta situación compromete la crianza y desarrollo de reemplazos, especialmente al considerar que estos animales representan el futuro de la explotación. Sin embargo, desarrollar reemplazos saludables que puedan parir alrededor de los veinticuatro meses de edad, es una labor que representa un alto costo en cualquier explotación ganadera, ya que, se debe invertir en alimentación, mano de obra, productos veterinarios y capital, entre otros rubros, por alrededor de dos años sin recibir ningún retorno económico.

Antes del año 1998, la Cámara Nacional de Productores de Leche utilizaba un modelo de costos para establecer el precio de la leche al productor, se valoraban todos los aspectos que estaban relacionados con el costo de producir leche para el productor. Con base en dicho modelo, se determinó que la crianza de animales para reemplazo correspondía al 25\% del costo total de producción de leche, y que cerca del 50\% de este lo representaba la alimentación y la mano de obra (Rivera, 2000).

En los Estados Unidos, Heinrichs (1993) reportó que, el desarrollo de reemplazos representa aproximadamente el 20\% del total de gastos de una finca lechera y es el segundo costo más elevado de la operación. Por su parte, León (2015) indicó que en Costa Rica, los costos de crianza representaban entre 8,98 y 16,54\% de los costos de producción de leche.

En un estudio realizado en los Estados Unidos, Gabler et al. (2000) reportaron que, el costo de criar y desarrollar animales del nacimiento al parto (veinticuatro meses) fue en promedio de $\$ 1124,06$. En un estudio más reciente, Heinrichs et al. (2013) determinaron que el costo total promedió los $\$ 1808,23 \pm 338,62$ en 44 explotaciones lecheras en el estado de Pensilvania, mientras que, en Costa Rica, Rivera (2000) indicó que los costos fueron de \$929,23.

El número de reemplazos que se crían y desarrollan en una explotación lechera, depende de muchos factores dentro del hato productivo y del hato de reemplazo. Así por ejemplo, dentro del hato de producción se indican factores como la tasa de descarte, el intervalo entre partos y la vida útil de las vacas, mientras que, en el hato de reemplazos se considera la tasa de mortalidad, edad a primer parto, tasas de descarte y eficiencia reproductiva, entre otros (Tozer y Heinrichs, 2001). 
Hay que tener presente que las operaciones ganaderas disponen de una serie de recursos y objetivos, de manera que la forma más económica de criar y desarrollar reemplazos, está determinada solamente por el análisis individual de los costos, y en Costa Rica se han realizado muy pocos estudios que indiquen el costo de producir animales de reemplazo. El objetivo del presente estudio fue determinar el costo económico asociado a la cría de reemplazos de lechería, desde el nacimiento hasta el primer parto.

\section{Materiales y métodos}

\section{Localización}

El estudio se llevó a cabo en la Estación Experimental de Ganado Lechero Alfredo Volio Mata de la Universidad de Costa Rica, durante el año 2016. Esta unidad está localizada a $1542 \mathrm{msnm}$ en Ochomogo de Cartago, donde la precipitación media anual es de $1502 \mathrm{~mm}$ (concentrada en la época de mayo a noviembre), la humedad relativa media es de $88 \%$ y la temperatura promedio anual de $17,9^{\circ} \mathrm{C}(\mathrm{IMN}, 2010)$. El ecosistema de la zona es considerado como bosque húmedo montano bajo (Vásquez, 1982).

\section{Procedimiento}

Para determinar los costos, se utilizó un total de cuarenta animales de la raza Jersey desde el nacimiento hasta el parto (veinticinco meses), agrupados en tres categorías de acuerdo con su edad: 0-3 meses, 3 a 16 meses y de 16 a 25 meses.

Como procedimiento normal de la explotación lechera, los animales al momento del nacimiento son separados de su madre, se les ofrece dos litros de calostro en las primeras dos horas de vida y se les repite la dosis seis horas después. Los animales luego son trasladados a cuadras individuales de cemento ( $2 \mathrm{~m} \times 2 \mathrm{~m}$ ) con cama de burucha, en donde permanecen hasta los tres meses de edad (doce semanas).

A partir del segundo día de vida y hasta los tres meses de edad, se ofrece leche íntegra a razón de cuatro litros diarios (2 1 am y 21 pm), y a partir del tercer día de vida se les comienza a ofrecer alimento balanceado, en pequeñas cantidades.

Una vez que los animales son destetados a los tres meses de edad, se trasladan a potreros bajo un sistema de pastoreo rotacional de pasto estrella africana, donde consumen el pasto a libre consumo, junto con $2,5 \mathrm{~kg}$ de alimento balanceado tipo desarrollo ofrecido de manera colectiva en el potrero, y sales minerales. Cuando los animales alcanzan el peso y edad óptima para el servicio (alrededor de los dieciséis meses), se inseminan y se les da seguimiento durante el periodo de gestación. En esta etapa los animales reciben una ración parcial mezclada que se prepara en un vagón mezclador autopropulsado, que contempla 1,3 kg de alimento balanceado y que es ofrecida de manera individual en un galerón de alimentación.

\section{Establecimiento de los gastos}

Para determinar los costos totales dentro de cada etapa, se llevaron registros en una hoja electrónica de todos los gastos en que se incurrió cada día y para cada animal, se consideraron todas las actividades que se llevan a cabo y que tienen que ver con alimentación, limpieza y desinfección, tratamientos veterinarios, fertilización de pasturas, mano de obra, plan sanitario, mantenimiento de instalaciones, etc., tal y como se detalla a continuación.

Leche íntegra: según el plan de alimentación a cada ternera se le suministraron cuatro litros de leche íntegra por día, que se multiplicó por el precio ofrecido por la planta industrializadora, en este caso de $₫ 310(\$ 0,56)$ por litro. 
Alimento balanceado: se llevó un registro diario de la cantidad de alimento ofrecido y rechazado para obtener así el consumo por animal, y este resultado se multiplicó por su valor comercial.

Mano de obra: consideró todo el tiempo utilizado para las diferentes labores como acarreo y ofrecimiento de la leche, cambio de camas, revisión del estado de salud de los animales, tratamiento de animales enfermos, ofrecimiento de alimento balanceado, areteado, tatuado, descorne, aplicación del plan sanitario, corrido y mantenimiento de cercas, preparación de la ración parcial mezclada, limpieza de las instalaciones, fertilización de las pasturas, lavado de utensilios, etc. El costo de mano de obra se consideró con base en un salario de $₫ 350000$ $(\$ 630,20)$, incluyendo las cargas sociales.

Plan sanitario: se consideró el plan establecido en la explotación para la prevención de enfermedades, y se llevó un registro de todos los tratamientos ejecutados durante el periodo de evaluación. En este caso se tomó en cuenta la dosis del producto utilizado multiplicado por su valor comercial.

Agua: se llevó un registro de la cantidad de agua utilizada para consumo y lavado de las instalaciones, que se multiplicó por la tarifa establecida por Acueductos y Alcantarillados de Costa Rica, reportada en $\phi 1405 / \mathrm{m}^{3}$ (AyA, 2016).

Electricidad: se estimó el consumo de electricidad utilizado por los bombillos, calentador de agua y funcionamiento de la cerca eléctrica. Este consumo se multiplicó por el costo de $\mathrm{kW}$, según la tarifa de la Compañía Nacional de Fuerza y Luz que, en este caso, fue de $₫ 104 / \mathrm{kW}$ hora (CNFL, 2016).

Viruta: se cuantificó la cantidad de burucha utilizada para las camas en la etapa de predestete y se multiplicó por el valor del kilogramo según el proveedor.

Semen: se utilizó el costo promedio de una pajilla de semen de acuerdo a las compras que se realizaron durante el año, y con base en los registros reproductivos, se utilizó un promedio de 1,7 inseminaciones por preñez.

Pasto: se determinó semanalmente el consumo de pasto mediante técnicas de muestreo conocidas, luego se cuantificó el contenido de materia seca en el laboratorio de bromatología de la Estación Experimental Alfredo Volio Mata y se multiplicó por $ф 14,6$, el costo por kilogramo de materia seca (Villalobos et al., 2013).

Heno: en algunas ocasiones se ofreció heno a los animales, por lo que, se estableció su consumo (considerando ofrecimiento y rechazo) y se multiplicó por su valor comercial.

Ensilado: los animales en la etapa de dieciséis meses en adelante consumen ensilado elaborado a partir de pasto Taiwán (Pennisetum purpureum), o sorgo negro forrajero (Sorghum almum), para determinar el costo de este rubro se utilizó un valor de $₫ 7,42$ por kilogramo de materia verde (Villalobos et al., 2015).

Minerales: se determinó la cantidad de minerales suministrada a los animales en la etapa respectiva y se multiplicó por su valor comercial.

Manejo sanitario: se siguió el plan sanitario establecido en la explotación, con desparasitaciones, aplicación de vitaminas y vacunación; se cuantificó la cantidad utilizada y se multiplicó por el valor del producto.

Gasolina: los animales a partir del mes 16 comienzan a recibir una ración parcial mezclada que se prepara en un vagón mezclador autopropulsado y que funciona por medio de gasolina. Se determinó el gasto diario y se dividió entre el número de animales que se alimentaron, luego se multiplicó por el costo de $\phi 579$ reportado para el litro de gasolina plus 91 por la Refinadora Costarricense de Petróleo (RECOPE, 2016).

Mantenimiento: se refiere al costo asociado al mantenimiento de instalaciones y equipo en la etapa de predestete, y al de las cercas en las otras dos etapas. No se consideró la depreciación de las instalaciones, ya que tienen más de treinta años de construidas.

Para estandarizar los costos de producción estimados para la crianza de terneras, se utilizó el valor promedio de $\Varangle 555,34$ obtenido entre la compra y venta del dólar estadunidense según el tipo de cambio anunciado por el Banco Central de Costa Rica para el 10 de noviembre del 2016 (BCCR, 2016). 


\section{Resultados}

\section{Costos de crianza ( 0 a 3 meses de edad)}

Los resultados de los costos de producción promedio de las terneras durante los primeros tres meses de vida se muestran en el Cuadro 1. Se determinó que el costo total promedio para criar una ternera a los tres meses de edad, bajo el sistema de crianza establecido en la explotación lechera de la Estación Experimental Alfredo Volio Mata de la Universidad de Costa Rica fue de $₫ 205948,00(\$ 370,85)$, esto representó un costo diario de $ф 2258,20(\$ 4,07)$.

Cuadro 1. Costo de producción de una ternera de cero a tres meses de edad en la Estación Experimental Alfredo Volio Mata. Cartago, Costa Rica. 2016.

Table 1. Cost of production of a dairy heifer from zero to three months of age in the Alfredo Volio Mata Experiment Station. Cartago, Costa Rica. 2016.

\begin{tabular}{|c|c|c|c|}
\hline Rubro & Colones & Dólares & Porcentaje \\
\hline Alimento balanceado & 27187,80 & 48,96 & 13,23 \\
\hline Leche íntegra & 104160,00 & 187,56 & 50,70 \\
\hline Agua & 220,21 & 0,40 & 0,11 \\
\hline Total de alimentación & 131568,01 & 236,91 & 64,04 \\
\hline Manejo sanitario & 1582,52 & 2,85 & 0,77 \\
\hline Burucha para camas & 28863,59 & 51,97 & 14,05 \\
\hline Electricidad & 7541,56 & 13,58 & 3,67 \\
\hline Mano de obra & 19942,32 & 35,91 & 9,71 \\
\hline Costo del semen & 14450,00 & 26,02 & 7,03 \\
\hline Mantenimiento & 2000,00 & 3,60 & 0,73 \\
\hline Costo total en la etapa & 205948,00 & 370,85 & 100,00 \\
\hline
\end{tabular}

\section{Costos del destete a los dieciséis meses}

Los costos establecidos para la etapa que va del destete a los dieciséis meses se presentan en el Cuadro 2 . En esta etapa, el rubro de alimentación representó el 86,19\% del gasto, del cual el alimento balanceado tuvo una ponderación del 69,80\%. El segundo costo que más influyó en esta etapa fue la mano de obra que representó un $5,04 \%$ de los costos totales de la etapa. El costo total para la etapa fue de $₫ 435980,57$ (\$785,07), lo que representó un costo diario de $\phi 1103,19(\$ 1,99)$.

\section{Costos de los dieciséis meses al parto}

Los costos establecidos para la etapa que va del servicio a los veinticinco meses se presentan en el Cuadro 3. En esta etapa la alimentación continuó siendo el rubro de mayor costo, con una participación del 77,56\%, del cual el 42,25\% correspondió a alimento balanceado, el siguiente costo de mayor influencia fue el de mano de obra con 
Cuadro 2. Costo de producción de una ternera de 3 a 16 meses de edad en la Estación Experimental Alfredo Volio Mata. Cartago, Costa Rica. 2016.

Table 2. Cost of production of a dairy heifer from 3 to 16 months of age in the Alfredo Volio Mata Experiment Station. Cartago, Costa Rica. 2016.

\begin{tabular}{|c|c|c|c|}
\hline Rubro & Colones & Dólares & Porcentaje \\
\hline \multicolumn{4}{|l|}{ Alimentación } \\
\hline Alimento balanceado & 304295,47 & 547,94 & 69,80 \\
\hline Heno & 5370,00 & 9,67 & 1,23 \\
\hline Pasto & 9602,60 & 17,29 & 2,20 \\
\hline Agua & 4185,78 & 7,54 & 0,96 \\
\hline Minerales & 52329,29 & 94,23 & 12,00 \\
\hline Total de alimentación & 375783,14 & 676,67 & 86,19 \\
\hline Plan sanitario & 19525,10 & 35,16 & 4,48 \\
\hline Vacunación & 5545,64 & 9,99 & 1,27 \\
\hline Electricidad & 1068,43 & 1,92 & 0,25 \\
\hline Fertilización & 10084,73 & 18,16 & 2,31 \\
\hline Mano de obra & 21973,53 & 39,57 & 5,04 \\
\hline Mantenimiento & 2000,00 & 3,60 & 0,46 \\
\hline Costo total en la etapa & 435980,57 & 785,07 & 100,00 \\
\hline
\end{tabular}

Cuadro 3. Costo de producción de una novilla de dieciséis meses de edad a primer parto, en la Estación Experimental Alfredo Volio Mata. Cartago, Costa Rica. 2016.

Table 3. Cost of production of a dairy heifer from sixteen months of age to first calving in the Alfredo Volio Mata Experiment Station. Cartago, Costa Rica. 2016.

\begin{tabular}{|c|c|c|c|}
\hline Rubro & Colones & Dólares & Porcentaje \\
\hline \multicolumn{4}{|l|}{ Alimentación } \\
\hline Alimento balanceado & 74547,20 & 134,24 & 42,25 \\
\hline Pasto & 31956,48 & 57,54 & 18,11 \\
\hline Ensilado & 17095,68 & 30,78 & 9,69 \\
\hline Agua & 10250,88 & 18,46 & 5,81 \\
\hline Minerales & 2991,20 & 5,39 & 1,70 \\
\hline Total de alimentación & 136841,44 & 246,41 & 77,56 \\
\hline Plan sanitario & 1091,12 & 1,96 & 0,62 \\
\hline Vacunación & 3774,44 & 6,80 & 2,14 \\
\hline Electricidad & 610,53 & 1,10 & 0,35 \\
\hline Fertilización & 5535,62 & 9,97 & 3,14 \\
\hline Mano de obra & 13862,42 & 24,96 & 7,86 \\
\hline Vagón mezclador & 12716,80 & 22,90 & 7,21 \\
\hline Mantenimiento & 2000,00 & 3,60 & 1,13 \\
\hline Costo total en la etapa & 176432,38 & 317,70 & 100,00 \\
\hline
\end{tabular}


una participación del 7,86\%. El costo total en esta etapa fue de $\phi 176432,38(\$ 317,70)$, lo que representó un costo diario de $\phi 642,74(\$ 1,16)$.

\section{Discusión}

El costo total de crianza ( 0 a 3 meses de edad) promedio obtenido en el presente trabajo, superó el costo reportado en el estudio realizado por Rivera (2000). Dicho estudio se realizó en siete fincas de lechería especializada en la Meseta Central de Costa Rica, donde se determinó que los costos de crianza de una ternera Jersey en el año 2000 desde el nacimiento hasta el destete a una edad promedio de 3,2 meses, fue de $\phi 121260,33$ $(\$ 218,35)$. Los rubros de alimentación, mano de obra y fertilización en el estudio de Rivera (2000), fueron los que más repercutieron sobre los costos de crianza.

En un estudio realizado por Elizondo-Salazar y Vargas-Ramírez (2015) se reportó un costo de $ф 221287,88$ $(\$ 398,47)$, en animales Holstein destetados a los cuatro meses de edad. Dichos autores indicaron que el rubro de mayor impacto en esta etapa resultó ser la alimentación con un $64,45 \%$, del cual un $24,57 \%$ correspondió al gasto de alimento balanceado, seguido por el concepto de lactoreemplazador y leche íntegra, los cuales representaron un 20,53 y $13,76 \%$, respectivamente. La mano de obra requerida para el cuido de los animales resultó ser el segundo costo de mayor importancia con una ponderación del 18,89\%.

En los Estados Unidos, Heinrichs et al. (2013) reportaron que, el costo de crianza del nacimiento al destete, realizado en promedio a los 57 días en 44 lecherías en el estado de Pensilvania, fue de \$247,38 \$86,21.

En el presente estudio, el rubro que más impactó fue también el de alimentación, que representó un 64,04\% del costo total de la etapa, del cual un 50,70\% correspondió a la leche íntegra. La utilización de burucha para las camas resultó ser el segundo rubro de mayor importancia con un 14,05\% y posteriormente, la mano de obra con un $9,71 \%$. En el estudio de Heinrichs et al. (2013) se determinó que, el costo del material utilizado para camas fue muy variable entre explotaciones; sin embargo, el costo fue muy alto y puede impactar significativamente el costo total en cualquier etapa donde se utilice.

Para poder comparar el costo total de crianza de una ternera del nacimiento al primer parto se utilizaron los datos reportados por Rivera (2000), que indicó que los costos fueron de $\phi 516042,61(\$ 929,23)$. En el presente estudio se obtuvo un costo total de $\not 818360,94(\$ 1473,62)$, lo que representó un incremento de $37 \%$ en un periodo de dieciséis años. Este incremento puede deberse a diversos motivos relacionados principalmente con el alza en el precio de los insumos utilizados, especialmente aquellos relacionados con la alimentación, que como se pudo observar en todas las etapas es el rubro que representó el mayor gasto. Con la información de este ensayo se determinó que la etapa del nacimiento a los tres meses, de los tres meses al servicio y del servicio al parto, representó un 25,$17 ; 53,27$ y $21,56 \%$ de los costos totales, respectivamente.

Al considerar los costos totales de las tres etapas, un 78,72\% correspondió a alimentación y un $6,82 \%$ a mano de obra. De los costos de alimentación, 63,03\% representó el gasto por alimento balanceado.

En los Estados Unidos, con la ayuda de un modelo dinámico de programación, Tozer y Heinrichs (2001) establecieron que el costo de criar y desarrollar una novilla en un hato de cien vacas en ordeño, con un intervalo de parto de trece meses, una tasa de descarte del $25 \%$ y una tasa de mortalidad de terneras del $10 \%$, fue de $\$ 1115,31$. Sin embargo, en otro estudio en el que analizaron datos de 44 explotaciones lecheras en el estado de Pensilvania, se determinó que el costo promedio fue de $\$ 1808,23 \pm 338,62$, y el costo de alimentación representó el $73 \%$ del total (Heinrichs et al., 2013). Los autores indicaron que el incremento tan sustancial se debió principalmente a los incrementos en los costos de alimentos y otros insumos. En otro estudio realizado en 49 explotaciones lecheras del estado de Wisconsin, se determinó que el costo de criar y desarrollar reemplazos del nacimiento al parto varió entre \$1595 y \$2935 (Zwald et al., 2007). 
Los costos de crianza y desarrollo de reemplazos van a ser muy diferentes en cada explotación, ya que cada una tiene diferente disposición de recursos y objetivos; sin embargo, es muy probable que un gran número de productores desconozcan dicha información y estén criando y desarrollando prácticamente todas las terneras que nacen en la finca, a pesar de que no tengan proyectado aumentar el número de vacas en ordeño, lo que repercute directamente sobre el flujo de caja, ya que los reemplazos requieren de una inversión económica importante, hasta que entran al hato de producción y no se hacen rentables hasta que pasan una o dos lactancias. Tomando esto en consideración, es importante que en las fincas se desarrollen solamente las terneras que van a ser necesarias para suplir el descarte o muerte de animales adultos. El número de terneras que deben desarrollarse en la finca, viene dado por la fórmula TH x (EPP/24) x TD x $(1+\mathrm{TMD})$, donde TH = tamaño del hato de producción, EPP = edad a primer parto, $\mathrm{TD}=$ tasa de descarte del hato, $\mathrm{TMD}=$ tasa mortalidad y descarte en reemplazos, según lo establecido por Bewley et al. (2010). Así, en una explotación con cincuenta vacas en ordeño, una edad promedio a primer parto de veintiséis meses, una tasa de descarte de vacas del 15\% y una tasa de mortalidad y descarte de reemplazos del $5 \%$, deberá desarrollar solamente alrededor de nueve terneras, lo que representa un 18\% del hato de producción (9/50). De esta manera, se le podrá dar prioridad a este grupo de animales y no habrá que invertir en todos, ya que si se considera, por ej., que un reemplazo en su primera lactancia produce 7000 litros de leche y se cuenta con una ganancia neta esperada $\notin 100(\$ 0,18)$ por litro de leche, se tardará alrededor de 1,17 lactancias para recuperar la inversión. Ahora, si la ganancia neta fuera de $\phi 50(\$ 0,09)$ por litro de leche, se tardará alrededor de 2,34 lactancias para recuperar la inversión.

\section{Conclusiones}

La crianza y desarrollo de terneras representa un rubro económico muy importante dentro de cualquier explotación lechera y conocer dicho costo, permitirá al productor criar y desarrollar solamente aquellos animales necesarios para reemplazar a las vacas de producción que abandonan el hato, ya sea por salud o por aspectos productivos o reproductivos.

\section{Literatura citada}

AyA (Acueductos y Alcantarillados). 2016. Costo del metro cúbico de agua. AyA, CRC. https://www.aya.go.cr/proveeduria/ SitePages/expedienteDigital.aspx (consultado 29 oct. 2016).

BCCR (Banco Central de Costa Rica). 2016. Tipo de cambio de compra y de venta del dólar de los Estados Unidos de América para el año 2016. BCCR, CRC. http://www.bccr.fi.cr. (consultado 10 nov. 2016).

Bewley, J.M., M.D. Boehlje, A.W. Gray, H. Hogeveen, S.J. Kenyon, S.D. Eicher, and M.M. Schutz. 2010. Stochastic simulation using @Risk for dairy business investment decisions. Agric. Fin. Rev. 70:97-125. doi:10.1108/00021461011042666.

CNFL (Compañía Nacional de Fuerza y Luz). 2016. Costo por KWh, tarifa menores o iguales que 3000 KWh. CNFL, CRC. https://www.cnfl.go.cr/servicios-residenciales-sr/tarifas-vigentes-sr (consultado 29 oct. 2016).

Elizondo-Salazar, J.A., y A. Vargas-Ramírez. 2015. Determinación del costo de la crianza de terneras desde el nacimiento hasta el destete en una lechería comercial especializada. Nutr. Anim. Trop. 9(2):1-10. doi:10.15517/nat.v9i2.21462

Gabler, M.T., P.R. Tozer, and A.J. Heinrichs. 2000. Development of a cost analysis spreadsheet for calculating the costs to raise a replacement dairy heifer. J. Dairy Sci. 83:1104-1109. doi:10.3168/jds.S0022-0302(00)74975-7. 
Gutiérrez, L. 2015. Análisis de la competitividad del sector lácteo costarricense: Visión de la Cámara Nacional de Productores de Leche. Pesentado en: XXI Congreso Nacional Lechero. Cámara Nacional de Productores de Leche, Heredia, CRC. 20-21 oct.

Heinrichs, A.J 1993. Raising dairy replacements to meet the needs of the 21st century. J. Dairy Sci. 76:3179-3187. doi:10.3168/ jds.S0022-0302(93)77656-0.

Heinrichs, A.J., C.M. Jones, S.M. Grays, P.A. Heinrichs, S.A. Cornelisse, and R.C. Goodling. 2013. Identifying efficient dairy producers using production costs and data envelopment analysis. J. Dairy Sci. 96:7355-7362. doi:10.3168/jds.2012-6488.

IMN (Instituto Meteorológico Nacional). 2010. Información climatológica de la zona de Ochomogo, Cartago. IMN, San José, CRC.

INEC (Instituto Nacional de Estadística y Censos). 2015. VI Censo nacional agropecuario. Resultados generales. INEC, San José, CRC.

León, H. 2015. Alternativas para la reducción de los principales costos de producción de leche en Costa Rica: alimentación y mano de obra. Pesentado en: XXI Congreso Nacional Lechero. Cámara Nacional de Productores de Leche, Heredia, CRC. 20-21 oct.

Madriz, J. 2014. Situación actual y perspectivas del sector lácteo costarricense: Visión de la Cámara Nacional de Productores de Leche. Pesentado en: XXI Congreso Nacional Lechero. Cámara Nacional de Productores de Leche, Heredia, CRC. 20-21 oct.

RECOPE (Refinadora Costarricense de Petróleo). 2016. Precios históricos consumidor final. RECOPE, CRC. https://www. recope.go.cr/precios-historicos-consumidor-final-1-16/. (consultado 29 Set. 2016).

Rivera, A. 2000. Determinación del retorno de la inversión en la crianza y desarrollo de novillas Jersey a primer parto en la zona de altura de la Meseta Central de Costa Rica. Tesis Lic., Universidad de Costa Rica, San Pedro de Montes de Oca, CRC.

Tozer, P.R., and A.J. Heinrichs. 2001. What affects the costs of raising replacement dairy heifers: a multiple-component analysis. J. Dairy Sci. 84:1836-1844. doi:10.3168/jds.S0022-0302(01)74623-1.

Vásquez, A. 1982. Estudio detallado de los suelos de la Estación Experimental de Ganado Lechero El Alto. Universidad de Costa Rica, San José, CRC.

Villalobos, L., J. Arce, y R. WingChing. 2013. Producción de biomasa y costos de producción de pastos Estrella Africana (Cynodon nlemfuensis), Kikuyo (Kikuyuocloa clandestina) y Ryegrass Perenne (Lolium perenne) en lecherías de Costa Rica. Agron. Costarricense 37:91-103.

Villalobos, L., J. Arce-Cordero, y R. WingChing-Jones. 2015. Costos de producción de ensilados de pastos tropicales elaborados en Costa Rica. Nutr. Anim. Trop. 9(2):27-48. doi:10.15517/nat.v9i2.21462

Zwald, A., T.L. Kohlman, S.L. Gunderson, P.C. Hoffman, and T. Kriegl. 2007. Economic costs and labor efficiencies associated with raising dairy herd replacements on Wisconsin dairy farms and custom heifer raising operations. Univiversity of Wisconsin-Extension, Madison, WI, USA. 